\title{
Cookie cloudy, cooked Google: ¡Al loro, bibliotecas en la nube!
}

Cookie cloudy, cooked Google: Be alert! Libraries in the cloud

\author{
Carlota Planas I SiLva \\ Sabatellini \& Associats, España \\ carlotaplanasisilva@sabateli.com
}

\begin{abstract}
Resumen
Las Bibliotecas tienen que vigilar cuando hagan el cambio a la Nube. La Nube es promocionada como un servicio gratuito. El concepto de gratuidad es irresistible a cualquier decisión de un consumidor. Esta es la estrategia de Google, también seguida por Facebook, Yahoo, Apple, entre otros. Sin embargo, ¿cuál es el coste?. Contenidos y privacidad son la moneda de cambio en la Nube, la cual, a su vez, está promocionada por instituciones públicas sólo como el remedio para superar la crisis. No importa si el remedio causa daños colaterales, como la vulneración de privacidad, contenidos o afectación del mercado de la libre competencia, con sus respectivas leyes. Las Bibliotecas tienen que estar alerta ante la Nube, pues tienen un importante legado para preservar, no solamente en términos de contenidos sino también de privacidad y valores. Si hay alguna curación, a la actual falta de legislación y jurisprudencia afín, debe ser a través de soluciones legales internacionales. Por desgracia, no parece que éste sea el pronóstico inmediato para hacer frente a la Nube. Google es realmente inmenso, en un gran logos, "dios", que sabe cada detalles de cualquier ser humano expuesto digitalmente en línea.
\end{abstract}

Palabras clave: Bibliotecas. Nube. Privacidad. Cambio digital. Derechos de autor. Google. Yahoo. Apple. Facebook. Derecho de la competencia. Desarrollo legislativo.

En el principio existía la Palabra y la Palabra estaba con Dios, y la Palabra era Dios. Ella estaba en el principio con Dios. Todo se hizo por ella y sin ella no se hizo nada de cuanto existe. (Evangelio según San Juan)

\section{De La "Nube" amiga a una "Nube" profesional: desvelando el gran disfraz}

En los tiempos que corren nuestros gobiernos están preocupados por defender las nacionalidades y las fronteras, sin ser conscientes de las grandes batallas que ya se están librando por parte de las grandes empresas en lo que será el nuevo ecosistema económico, social y cultural del siglo XXI, la Nube.

\begin{abstract}
Libraries have to be care of the cloud computing shift. Cloud Computing is promoted as a free service. The free concept is irresistible to consumer's choice. Mainly, it is Google's strategy as well followed by Facebook, Yahoo, Apple, among others. Nevertheless, at what cost is the Cloud free? Contents and privacy are the means for trading in the Cloud, which is promoted, as well, by public institutions just as a key-remedy to overcome the crisis. No matter if the remedy brings collateral damages, such the breach of privacy, copyright and competition laws, among other ones. Because such laws are old-fashioned, there is a lack of legislation and case law to rule cloud computing. Libraries have to be awared of cloud computing: they have an important heritage to preserve. Not only the content, but also the privacy and values. If any heal has to be implemented, it cannot be by the governments of each country, but it has to be through international legal solutions. Unfortunately, this does not seem to be the immediate forecast. Google is already certainly a huge, a huge "logos", "god", which knows every single detail of being digitally-exposed human.
\end{abstract}

Keywords: Libraries. Cloud. Privacy. Digital change. Copyright law. Google. Yahoo. Apple. Facebook. Competition law. Legislative development.

Como dice Le Crosnier (2008), estas empresas no tienen apetito de frontera, tienen avidez de consumidor-cliente. Estamos ante una industrialización de los servicios a través de la informática, lo cual supone un cambio de paradigma, esto es los recursos informáticos como un suministro de gas, de teléfono... bajo la divisa "pay as you go". Las grandes empresas en la Nube buscan economías de escala.

El término "Nube", "Cloud Computing" puede ser nuevo como encuñadura, pero la Nube existe desde hace mucho tiempo y lo venimos empleando y la conocemos desde casi los inicios de Internet, léase Yahoo Mail, Gmail de Google, Facebook, Youtube, Flickr, Microsoft Office Web Apps.... Todos ellos son ejemplos de servicios informáticos en la "Nube", virtuales. Es decir, se 
ha pasado de una instalación física, material y local, a una instalación virtual y global.

La "Nube" hasta la fecha ha mostrado su cara más amable y se ha vendido con su cara más simpática, con un objetivo: crecer con masa crítica. Al buscar economías de escala, la tecnología y la inversión de la "Nube", así como su repercusión es más sostenible. Se acuñan términos muy "cool" como: "Green Tech" o "Zen Tech", otros términos también responden a éstas nuevas premisas de vocablos: reserva de recursos o multiposesión por parte de varios clientes o usuarios simultáneamente; accesibilidad en cualquier momento, en cualquier sitio y casi de cualquier forma - PC, Applets, móviles, televisión con Internet...-: todos son términos "cool" para las "new tools".

\section{La velocidad expansiva de La "Nube"}

La migración hacia la "Nube" va en aumento. Cada vez son más las empresas, instituciones públicas, privadas $\mathrm{y}$, por supuesto particulares, que están entrando en esta "Nube". Una "Nube" que cada vez es más perfecta a nivel tecnológico. Y las empresas "Nube" están facturando más que las empresas industriales; y sus disputas para posicionarse y conseguir su cuota de mercado son cada vez más feroces. El año pasado Google y Microsoft tuvieron varias disputas en relación a la adjudicación de contratos administrativos de varios estados de los Estados Unidos, para la gestión de correo electrónico y otros servicios.

En 2010, en Estados Unidos, 240 millones de personas usaban regularmente Internet. La actividad comercial en Internet de aquel año fue de 170 billones de dólares. Tanto la Administración Americana como la Administración de la Unión Europea consideran la "Nube" como un sector estratégico para hacer frente a la crisis, una nueva tierra de oportunidades, totalmente virtual (Kroes en Davos, 2011; Vanacek, 2011).

\section{Las distintas categorías de Nubes}

En este artículo nos vamos a concentrar en la Nube conocida como SAAS, si bien existen varias categorías de Nubes, según el criterio de clasificación que se adopte.

La "Nube" es Internet y, en particular, la capacidad que tiene ésta de procesar y almacenar cosas, dentro de esta definición amplia se pueden categorizar distintas modalidades de "Nubes" (Fundación de la Innovación, Bankinter, 2010):

1. Las infraestructuras como servicios (IAAS). Léase los servicios web de Amazon que permiten el almacenamiento, bases de datos escalables, servidores privados virtuales y servicios de soporte que se contratan bajo pedido, sea por hora o megabyte. New York Times es cliente de Amazon y utiliza la infraestructura S3 de Amazon, Bungee.

2. La plataforma como servicio (PAAS) es el siguiente nivel de nube. En esta nube las empresas pueden modificar sus propias herramientas: prototipos, desarrollo, pruebas, alojamiento. Entre otros actores se encuentran Google Application Engine, SalesForce.com, Microsoft Azure, Facebook.

3. El software como servicio de aplicaciones (SAAS). Procede del antiguo acrónimo Application Service Provider (ASP). Los elementos que lo caracterizan son el alojamiento y suscripción, como por ejemplo Google Docs, Application Store.

4. El software como servicio (SAS): esto es, utilizar servicios accediendo a través del buscador, léase ADP.

Otro modo de categorización es a través de determinar quién es el titular de la "Nube": Nube Privada, Nube Pública, Nube Híbrida o Nube gubernamental (g-cloud, government cloud)

En definitiva, esta categorización describe modelos a la carta para la asignación y consumo de recursos informáticos, siendo mucho más amplio el concepto que el simple SAAS, pues incluye tanto aplicaciones, servicios como hardware, y deberán tenerse en cuenta muchos aspectos, compartiendo esta opinión Larry Ellison, Presidente y Fundador de Oracle (2008) y Richard Stallman (2008), el fundador de GNU pionero en el código abierto.

Sin embargo, a pesar de todas estas clasificaciones, uno de los problemas que plantea el concepto "Nube" es su definición técnicojurídica, como se verá más adelante.

\section{La "Nube": en busca de réditos digitales}

Esta cara amable, simpática que prodiga una "Friendly Cloud" frente una "Evil Cloud", crece más aún por la facilidad en poder acceder a los servicios y productos; pues la mayoría son gratuitos para los usuarios, a los cuales no les importa donde se ubica la información, ni la tecnología, ni mucho menos quién la maneja; extremos que deberían importar cuando se está hablando de contenidos, de datos personales, de ideología...

A las bibliotecas sean públicas o privadas se les ofrece la "Nube" como un hábitat que puede ser 
de lo más natural para ellas, pero tienen que conocer todos los puntos débiles y críticos de las "Nubes", pues no deja de ser una tentación la manera en que se ofrecen estos productos y servicios, y también es muy rápida la velocidad en que las condiciones de contratación se cambian o se pueden cambiar.

Pues no debemos olvidar que ni Google, ni Apple, ni Microsoft, ni Amazon, entre otras, son entidades sin ánimo de lucro, sino que son empresas con inversores, socios que buscan rentabilidades. Así, por ejemplo, si Hewlett-Packard ha comprado Electronic Data Systems (EDS), para posicionarse en la "Nube", por una buena cantidad de dinero, será para buscar un beneficio a corto o medio plazo. Estos extremos hay que tenerlos en cuenta cuando las bibliotecas emigran a la "Nube".

\section{El maná de la Nube para las bibliotecas con sus cautelas}

La "Nube" SAAS puede resultar muy beneficiosa para las bibliotecas. A nivel tecnológico, uno no se debe preocupar por la tecnología y, en particular, por la mejora constante de la tecnología subyacente. No se depende de una tercera empresa contratista o proveedora de servicios; de forma que las bibliotecas se pueden dedicar a ofrecer sus verdaderos servicios a los usuarios con respecto de sus fondos.

La "Nube" puede ser un verdadero océano de divulgación de contenido: construcción y actualización de los fondos, ordenación, nuevos servicios, acceso al contenido con una mayor facilidad, buena capacidad para almacenar la información. A nivel económico se pasa de unos costes fijos y directos, a unos costes variables e indirectos, es decir, se compran servicios o productos "Nube" cuando es necesario y útil. Lo cual para las bibliotecas es de lo más óptimo para reducir los costes directos y fijos.

A nivel de comunidad de contenidos también puede resultar muy beneficioso a los efectos de utilizar una tecnología común para alcanzar acuerdos de divulgación con terceras bibliotecas pues se parte de una misma plataforma o aplicaciones (Teregowda, Urgoankar y Giles, 2010; Goldner, 2010; Malpas, 2011; Sanchati y Kulkarni, 2011).

Todos estos beneficios deben sopesarse y valorarse frente a las desventajas que, quizás, son menos visibles: las jurídicas; o, incluso, pueden considerarse como "daños colaterales asumibles", pues el fin principal está salvaguardado a un coste cero o casi cero (Renfrew, 2012). Sin embargo, el señuelo de coste cero o casi cero puede perturbar una decisión neutral sobre los contenidos.

\section{Los grandes nubarrones legales de las "Nubes"}

El gran entusiasmo inicial de la "Nube" se desvanece en gran parte, cuando se profundiza en esta "Nube" desde un punto de vista legal. En primer lugar, porqué hay que observarla desde un punto de vista multinacional, pues están involucrados muchos países: un cliente en un país $A$ utiliza un SAAS por un proveedor en un país $B$, el cual adquiere la infraestructura en un país $C, D$ y/o $E$ dependiendo del precio, básicamente, pues no olvidemos que al usuario final también se le debe ofrecer a un coste cero o casi cero.

Más aún, una "Nube" se puede construir y destruir sin que nadie sepa dónde está ni quién verdaderamente la tiene y qué hace con ella, y menos aún dónde ha ido a parar su información.

La seguridad, privacidad, accesibilidad de contenidos y datos personales, posición abusiva en el mercado son los grandes y graves problemas de la "Nube"; y no todas o casi ninguna de las "Nubes" da unas garantías plenas con respecto a estos grandes problemas, en particular las "Nubes" gratuitas o casi gratuitas.

No se trata de especular en posibles casos, sino en verdaderos casos que se están dando en la actualidad fruto de una ausencia de regulación específica a ese estado que es la "Nube" y que dé respuestas a los problemas que genera la "Nube". Tanto la legislación como la jurisprudencia son más propias de tiempos pretéritos. La "Nube" y su crecimiento vertiginoso han pillado a los reguladores y a la Justicia sin los instrumentos adecuados, tanto a nivel nacional como internacional.

En Estados Unidos hay iniciativas para intentar regular las actividades en la "Nube", a través de un código de buenas prácticas así como los aspectos críticos de la "Nube", con el fin de concienciar sobre las actividades en la "Nube" (véase la Cloud Security Alliance). Asimismo, en el Informe de la Unión Europea, sometido a consulta pública, sobre la "Nube" (2011), se pone de manifiesto que hay que establecer unas bases, guías o recomendaciones. Es más, en Estados Unidos ya ha habido alguna decisión sobre las implicaciones legales de la "Nube" (véase Oregon v. Bellar), en donde un $69 \%$ de residentes americanos están conectados a la red, y al menos a una "Nube". 


\section{Los contratos con los proveedores de servicios y productos en la Nube: el inicio de todos los problemas}

La problemática que genera la contratación con proveedores en la "Nube", es que estamos ante contratos de Adhesión con cláusulas estándares iguales para todos, pero con una total y absoluta exclusión de responsabilidad por parte de los proveedores o minimización de los remedios de reparación si hay algún problema. Se parten de sistemas de contratación propios del sector informático, como los "ClickWrap Agreements".

Generalmente, los proveedores serán absolutamente reticentes a negociar obligaciones más gravosas, aunque sea para cumplir con la normativa vigente de protección de datos personales y contenidos, entre otros, pues es incompatible con su modelo de negocio, el cual es muy "barato" o, supuestamente, casi "gratuito".

Firmar este tipo de contratos significa que no se controla nada de los servicios que se ofrecen, ni cómo se ofrecen ni cuando se ofrecen, pues existe la doble excusa: la gratuidad y la aceptación.

El contrato con un prestador de servicios en la "Nube" debería tener presente: (i) ubicación de los datos, dónde se encuentran almacenados; (ii) garantías de seguridad y toma de medidas de seguridad acreditables; (iii) copias de seguridad para la recuperación de los datos frente a cualquier desastre o incluso migración de datos, especialmente saber sus costes; (iv) reconocimiento expreso de la propiedad de los datos y/o contenidos que son cargados en la "Nube" y datos secundarios, incluyendo métodos, que se generan por la interactuación en la "Nube"; (v) garantías de que las copias de datos personales serán eliminadas de la plataforma una vez terminado el contrato, en cualquier caso estableciendo plazo para la eliminación; debería existir un nivel de servicios (LSA) que debería revisarse también, periódicamente; y (vi) se deberían evitar cláusulas relativas a una revisión unilateral de las cláusulas del contrato por parte del proveedor (Araiza, 2011).

Sin embargo todas estas cautelas no se tienen en cuenta por los usuarios, sean más grandes y/o más pequeños, pues la naturaleza gratuita de la oferta es demasiado tentadora para desaprovecharla. De forma que el firmanteadherente ni tan solo repara en la legislación y jurisdicción a la que se someten estos contratos, en caso de incumplimiento, lo cual puede suponer entrar en otro problema.

\section{8. ¿En la "Nube" qué ley aplicamos? Depende como se defina la "Nube"}

En la actualidad, los jueces no entienden qué es la "Nube", y por ello hay resoluciones absolutamente distintas en diferentes países de la Unión Europea. Prueba de ello son las resoluciones adoptadas en España y en Reino Unido.

En España, de conformidad con la Ley de la Sociedad de la Información, la Audiencia Provincial de Madrid, en el caso de Google y el contenido de las páginas web de PR Noticias Telecinco y "Aquí hay Tomate", reconoció que quedaba acreditado que Google disponía de oficinas en España es decir "bien disponga, de forma continuada o habitual, de instalaciones o lugares de trabajo, en los que realice toda 0 parte de su actividad" y por ello, la Audiencia Provincial de Madrid (Sentencia Audiencia Provincial de Madrid, 2010, JUR/2010//133011) entendió que Google era un prestador de servicios en una red de comunicaciones facilitando datos en España.

En cambio, en el Reino Unido se trató de forma completamente distinta el caso Football Dataco v. Sportradar Gmbh, Football Data, un compilador de datos sobre fútbol. Sportradar está en Alemania y Austria y tiene hospedados los resultados de búsqueda en estos respectivos países. Football Dataco demandó a Sportradar por copiar estos contenidos en el Reino Unido. El juez entendió que la normativa aplicable era la normativa de transmisión de contenidos vía satélite, esto es, el lugar donde el contenido es introducido bajo el contrato de la persona que hace retransmisión, Alemania, en vez de donde se cargaban los resultados, Reino Unido. Así, según la mencionada sentencia, un servidor no es equivalente a "uplink site". Por ello el sitio donde se hace accesible es el sitio donde alguien descarga y no donde está ubicada la información. Con este tipo de razonamiento uno se puede encontrar con que nunca se le aplique la legislación donde tiene ubicado el servicio (Indegment of Floyd, 2010).

De forma que la definición de "Nube" es muy importante para poder saber cuál es la legislación aplicable. 


\section{Privacidad y protección de datos: ¿Ellos saben de tus usuarios y tú qué sabes de ellos? ¿Y los usuarios conocen lo qué tu haces con ellos?}

\author{
9.1. Las Huellas que dejamos cuando \\ navegamos
}

Todos nosotros al navegar o entrar en las "Nubes", simplemente a curiosear, dejamos un rastro que a las empresas como Facebook, Google, Apple, Yahoo... les interesa disponer durante mucho tiempo, para ofrecer estos datos a los anunciantes. Incluso, han aparecido empresas especializadas en el manejo, eliminación de lo que técnicamente se llama e-reputación de las personas físicas y jurídicas.

El Gobierno Americano a través de Federal Trade Commission y Department of Commerce emitió sendos informes en Diciembre de 2010 sobre la Red. Se exponía de forma muy clara que cada paso que se hace en la Red es observado, cada click almacenado, tanto de entidades privadas como públicas y cada respuesta es "convected" por atentos buscadores e influenciadores de conducta. Más aún la Federal Trade Commission ha emitidio un Informe en relación a la privacidad y recolección de datos de menores para la descarga de aplicaciones en la "Nube", tanto en el sistema operativo Android como del sistema operativo IOS (Children's Online Privacy Protection Act).

En definitiva, el principio de transparencia y elección propio de la Red y promovido por los grandes buscadores - "transparency and choice" - se ha cambiado por otro - "take it or leave it". En la actualidad, en ningún caso, la "Nube" es un territorio virgen (Multh, 2009; y en la misma línea Meuli, Finn, desde una óptica ética de protección de datos).

\subsection{Google: Paradigma Emergente de Logos Total}

Google Inc. puede ser en la actualidad la empresa privada con más información, datos y contenidos del mundo. En 2008, Google Inc., tenía un millón de anunciantes en línea, frente a los 300.000 de Yahoo y los escasos 75.000 de Microsoft. Apple ha pasado de ser una boutique tecnológica a un "trendding-setting", de facturar en 2001 cinco billones de dólares a 108 billones en 2011 , con un valor de mercado de 510 billones de dólares, más que Microsoft y Google juntas. Yahoo demandó a Google antes de su salida a bolsa y consiguió que Google le diera acciones, ahora también lo ha intentado con Facebook por la violación de patentes relativas a la privacidad y publicidad (véase El Periódico, 2012). Todas estas cifras no son gratuitas, es para dimensionar la realidad de la "Nube", pues al ser todo inmaterial, su visibilidad es inexistente, pero en cambio la confianza depositada por el consumidor-cliente es total, sin ver ni tocar. Un verdadero acto de fe.

En 2008, Yahoo y Google tuvieron que abandonar el acuerdo de publicidad, después de la advertencia del Departamento de Justicia Americano, que los amenazó con una demanda, pues entendía que se podían convertir en colaboradores, en vez de competidores. En 2009, tres empresas - Google, Yahoo y Apple-fueron nuevamente examinadas, pues habían alcanzado un acuerdo entre ellas para no contratarse los altos cargos. En 2010, se les requirió de nuevo, pues Apple y Google estaban compartiendo dos consejeros de sus respectivos consejos de administración.

Por otra parte, el cambio de política de privacidad de Google en marzo de 2012, a raíz de la nueva herramienta Search + , la cual se parece más a la de Facebook, no permite el "opting out". Así, la Política de Privacidad de Google es que no hay privacidad.

Tanto las Autoridades Americanas como Europeas están pidiendo explicaciones a Google sobre el cambio de Política de Privacidad, Política que forja ella y decide ella el nivel de seguridad, así véase Reuters (2012). Esta situación no es nueva, anteriormente ya había habido otros casos.

\subsection{Google y sus excusas tecnológicas para expandir su Logos}

Google, como ya hizo en la digitalización masiva de libros, adopta una actitud maliciosamente inocente frente a prácticas que afectan a la privacidad de las personas para seguir su negocio de forma expansiva, véanse varios ejemplos:

1. En 2009 el sistema de correo electrónico falló durante 3 horas y 113 millones no pudieron acceder a sus correos que estaban en Google Docs. La respuesta de Google fue "Ups!!, lo sentimos estábamos mejorando".

2. En el caso Buzz, otra plataforma de Google que utiliza de forma alternativa, estaba también investigando datos. La justificación de Google, nuevamente: una sonrisa y "Ups!! estábamos recopilando información para la investigación y análisis".

Otra marca blanca de Google es Searchmash.com, lo cual le permite con nuevos nombres e interfaces distintos seguir anali- 
zando conductas y disponer de más datos personales. El usuario solamente sabe que detrás está Google, si tiene la precaución de leerse la política de privacidad, lo cual casi nunca se hace.

A finales del año pasado, 2011, en Estados Unidos, un estudiante de la Universidad de Standford, J. Mayer encontró a Google y otras tres empresas de publicidad en línea que estaban pasando por encima de la configuración predeterminada del navegador de Safari para poder rastrear los hábitos de navegación de los usuarios, y, así, ofrecer una publicidad más eficiente. Mayer denunciaba a Google pues, de forma unilateral, había decidido eliminar los términos de privacidad y restricciones con respecto de productos de Google, esto es, las "first party cookies" que tienen tanto Facebook y Google, las cuales no se tienen que aceptar cada vez que se visitan las páginas en cuestión. En este caso, Google se excusó con "Oops, sorry, that was a mistake, we did not know we were doing that..." Es decir, Google va más allá y sus condiciones de privacidad lo avalan:

We use cookies to improve the quality of our service by storing user preferences and tracking user trends, such as how people search.

3. En sus condiciones de privacidad Google dice que no comparte con terceros los datos personales, pero, en realidad, no es verdad, pues sí que lo comparte con empresas subsidiarias, afiliadas y otras empresas de confianza, incluso, por supuesto, aquellas empresas que compra. Así, Youtube es un ejemplo de cómo acceder a más información mediante la compra.

4. Gmail siempre conserva copia aún cuando se borre toda la información. Todos los correos son escaneados y guardados.

Google Maps y Google Search son capaces de guardar todas las búsquedas y saber qué has estado mirando. Prueba de la facultad de imperium de facto de Google, es que tiene una nueva política de privacidad desde el 1 de marzo de 2012, por el momento, sin que ninguna autoridad gubernamental de ningún país haya podido impedir su eficacia o sancionarla.

\section{Las Herramientas Legales de Estados Unidos frente al Logos}

\author{
10.1. La Stored Communications Act y Electronic \\ Communications Privacy Act, inservibles
}

La ley aplicable para todo el tema de la "Nube" es una ley de 1986, ampliada por la Electronic Communications Privacy Act, Stored Communications Act. De conformidad con esta normativa, se establece que la privacidad requiere el almacenamiento electrónico, que debe ser la única razón en virtud de la cual el usuario envía datos al proveedor de la "Nube". Cuando la información es compartida por el proveedor de la "Nube" para ofrecer anuncios, la ley no se cumple. Sin embargo, si hay consentimiento por parte del usuario, la ley no se aplica, así que el usuario deberá tener muy en cuenta el acuerdo que firma o acepta, extremo que ya hemos apuntado en este caso (18 V.S.C \# 2701 a 2712).

Por consiguiente en el caso de Google, con su Gmail, es muy fácil el consentimiento, pues el paradigma es de acuerdo total y sin limitación

The Gmail filtering system also scans for keywords in users' e-mails which are the used to match and serve also. When a user opens an e-mail message computers scan the text and then instantaneously display relevant information to the text of the message $[\ldots]$ by submitting, worldwide royalty free, and non-exclusive license to reproduce, adapt, modify, translate, publish, publicly perform, publicly display and distribuye any content which you submit, post or display on or through (Google's services).

10.2. La Cuarta Enmienda de la Constitución Americana: invisible frente a la "Nube"

La posibilidad de uso de la Cuarta Enmienda de la Constitución Americana relativa a la privacidad, la jurisprudencia americana solamente la aplica frente a las instituciones públicas, y no a las instituciones privadas. De forma que las instituciones privadas son libres de invadir la privacidad en su beneficio, incluso de compartir los datos con instituciones públicas, es decir, defraudar la Ley.

Así, en relación a la Cuarta Enmienda, en el caso United States vs. Miller, el Tribunal Supremo negó eficacia a la Cuarta Enmienda, pues la información bancaria que se solicitaba a los bancos no era privada, sino de una empresa.

La Cuarta enmienda parte de dos principios: (i) necesidad y (ii) expectativa. Así, en origen, se entendió la necesidad de usar el servicio postal y el teléfono como algo sin alternativa; ahora, lo mismo se puede ya predicar con Internet $y$, con la "Nube". Los Tribunales americanos han con- 
siderado que la expectativa de que los usuarios y su correo postal y conservaciones estuvieran expuestas a la no privacidad era limitada.

El Congreso adoptó estos mismos criterios para defender la Third-party Disclousure Doctrine para comunicaciones electrónicas y servicios electrónicos remotos.

Por consiguiente la jurisprudencia americana es difícil que aplique la cuarta enmienda en la Red. En definitiva y jugando con la terminología anglosajona, dado que estamos en su territorio, a los efectos de exposición de este artículo, se puede concluir: Google for free but no with freedom for you.

\subsection{La Pasividad de la Jurisprudencia} Americana bajo el amparo de la normativa

Con la normativa actual americana, los tribunales americanos no entran a valorar las condiciones de privacidad y, menos, especular sobre las probabilidades de uso incorrecto de los datos. De forma que un proveedor en la "Nube" tendrá total libertad para usar la privacidad y datos personales a su antojo (Robinson, 1995). Así, en el caso Marrero-Hernandez v. Esso Standard Oil Co., dijo que, si las partes optaban en usar recursos tecnológicos para almacenar información privilegiada, también deberían tomar las medidas de protección necesarias.

10.4. La fuerza de la sociedad civil americana frente la capitalización de la privacidad de Google

La sociedad civil americana se ha dado cuenta que la actual situación va en regresión a sus derechos y sus individualidades; por eso, empieza a moverse y manifestarse en contra de esta legislación y jurisprudencia americana, que lo único que provoca es el hastío de los ciudadanos, pues supone invertir en tiempo y recursos económicos, lo cual es complejo y, al final uno se cansa (Gellman, 2009; Weber, 2011; Week, 2012).

Ejemplos de la iniciativa privada son la red www.donotrack.us, impulsada desde la Universidad de Standford, y organizaciones privadas sin ánimo de lucro como World Privacy Forum, que están luchando para que haya un cambio en la legislación y, por ende, en la Jurisprudencia Americana.

Sin embargo, en fecha reciente, 30 de Noviembre de 2011, se ha promovido la Cyber Intelligence Sharing Protect in Act (CISPA). Ésta trata de defender a gobiernos y compañías frente a los ciberataques, pero, en realidad, la ley permite la posibilidad de eximir de responsabilidad a aquellas empresas privadas que faciliten información sobre violaciones de derechos, en particular, de copyright. A cambio, van a seguir disponiendo de una base de datos personales privatizada, tanto Google como el resto de empresas de Internet.

\section{En España, haciendo malabarismos con la legislación y jurisprudencia frente al Logos Google con breve mención al derecho al olvido}

\subsection{La actuación activa de la Agencia de Protección de datos Española}

Ni la perseverancia de la Agencia de Protección de Datos Española ni la LORTAD ni la LOPD son suficientes para atender los retos de la "Nube". Ha sido a través de la Agencia Española de Protección de Datos que se han venido delimitando, a través de sus resoluciones, criterios para tutelar la procedencia del derecho de cancelación y oposición en servicios de búsqueda. Las reclamaciones recibidas en este ámbito han tenido su origen en la indexación y recuperación por buscadores de Internet de datos personales contenidos en boletines oficiales y ediciones digitales de medios de comunicación (Lombarte, 2010). En una de las más señaladas se dispuso así (Resolución de la Agencia de Protección de Datos, R//00896/2011)

[...] debemos indicar que la Ley no dispone que los datos personales del reclamante figuren en los índices que utiliza Google para facilitar al usuario el acceso a determinadas páginas, ni tampoco dispone que figuren en las páginas que Google conserva temporalmente en memoria "caché" [... Por lo que ], procedía la exclusión de los datos personales de la reclamante de los índices elaborados por Google, así como la eliminación de las imágenes que aparecen en la página reclamada del Blogger.

Es verdad que el principio inicial en la "Nube" es la libertad de expresión, y así lo ha entendido la Jurisprudencia del Tribunal Constitucional, frente a otros derechos, siempre que los personajes en cuestión sean de relevancia pública y atendiendo a la veracidad de la información facilitada.

\section{La propia Audiencia Nacional ha reconocido que}

ninguna objeción puede hacerse a la finalidad que persigue el derecho de la información veraz, pero dicho derecho fundamental no es un derecho absoluto, sino que hay que ponerlo en relación a otros derechos fundamentales, como lo es en este caso, el derecho fundamental a la protección de datos $[\ldots]$.

A 31 de marzo de 2012, se ha publicado una modificación sustancial relativa al uso de co- 
okies en páginas web. Hasta ahora era posible usarlos con el simple consentimiento tácito. Con esta nueva normativa, las cookies necesitarán el consentimiento expreso e informado del destinatario para poderlas implementar. Sin ánimo de extendernos, nos remitimos a lo ya expuesto sobre Google, en concreto, en el apartado 9.1. de este artículo.

\subsection{El derecho al olvido defendido por la} Jurisprudencia española

La realidad es tan dinámica que, hace unos días, la Audiencia Nacional Española elevaba una cuestión prejudicial al Tribunal de Justicia de la Unión Europea, en base a varios casos (véase el Auto de la Audiencia Nacional, 2012), afectando todos ellos a Google y se quiere determinar "la actividad de los buscadores en Internet" y "su sometimiento a la normativa en materia de protección de datos": si los buscadores que indexan la información están realizando un tratamiento de datos personales, y si la protección de datos incluye el derecho al olvido. La posición de Google en este procedimiento parte del concepto de libertad de expresión. Así CarriIlo (2009) dice

No podemos ser los censores de Internet. Nosotros nos limitamos a reflejar de forma exhaustiva el contenido en Internet, lo que existe de verdad en la Red, lo que esperan nuestros usuarios [...] Nosotros queremos cumplir [...] y eliminar los enlaces y borrar del índice y de la memoria caché, siempre y cuando la fuente original también los retire.

Rosen (2012), por su parte, presentan la visión americana de la legislación europea, en definitiva, el derecho a enlazar que no el derecho del olvido. Es más, en el caso de las bibliotecas, si éstas son de naturaleza pública, deben ser consideradas una administración pública y les será de aplicación el Plan Nacional de Seguridad.

Lo que se desprende de la filosofía Google es que al haberse montado el negocio en base a la gratuidad es muy difícil eliminar in eternum las huellas, rastros, rostros, noticias de uno o alguien.

\section{La futura reglamentación europea de privacidad limitada: mercado único digital y privacidad}

Según la Comisaria Europea de Justicia, Redding, "Dios perdona y olvida, pero la web nunca”. La futura reglamentación europea sobre privacidad parte de los siguientes principios: (i) La carga de la prueba recaerá sobre la empresa y no sobre el usuario. (ii) Privacidad por defecto. (iii) Derecho de portabilidad, derecho de obtener una copia de los datos almacenados por una red social y la libertad de trasladarlos a otra. (iv) Las compañías deberán notificar en el plazo de 24 horas, tanto interesados como autoridades de protección de datos. (v) Sanciones de un millón de Euros o el $2 \%$ del volumen de negocios mundial de la compañía. (vi) Información de cómo se está controlando su uso en Internet para dirigirles publicidad después de haber consultado las páginas.

En definitiva, la sensibilidad Europea es distinta a la Americana, y en este caso, si finalmente se aprueba y entra en vigor esta normativa, se deberá revisar el Acuerdo de Safe Harbour vigente, que existe entre Estados Unidos y la Unión Europea.

\section{La "Nube" relativiza el valor de los contenidos, derechos de autor}

13.1. La jurisprudencia americana atrapada por una legislación absoluta: Estados Unidos, Digital Millenium Copyright Act (DMCA)

En la sección 512 de la DMCA, se limita la responsabilidad de los proveedores de servicios, si se cumplen las condiciones:

[...] service providers are not liable for infringement of copyright by reason of the provider's transmitting, routing, or providing connections for, material through a system or network controlled or operated by or for the service provider or by reason of the intermediate and transient storage of that material in the course of such transmitting, routing or providing connections.

Por ello, en agosto del año pasado, un juez federal del Estado de Nueva York entendió que no había infracción de los derechos de copyright en los servicios de alojamiento de música MP3, pues el servidor no promovía la infracción, "to induce infringement", mutatis mutandis Google Music y Amazon Cloud Drive "Alternatively cloud lockers agnostically host whatever music wants to upload". Previamente, la justicia americana había tenido ya la oportunidad de posicionarse de forma similar con un SAAS en el caso Veoh (véase Universal Music Group v. Veoh), lo cual se podría también trasladar a PAAS y IAAS. Estos servicios, por otra parte, tienen menos posibilidades de infringir la DMCA, pues no tienen un control interactivo tan amplio sobre el contenido: sólo en el caso que haya una inducción a la infracción, como así se insinuó en el caso Grokster.

Efectivamente, en el caso Grokster se sugirió que varias posibles actividades podrían conllevar infracción de la DMCA, como por ejemplo publicitar un mensaje promoviendo la infracción de contenido (Melzer, 2011). 
La doctrina americana sobre el copyright se rebela en contra de la posición de la jurisprudencia americana, si bien la culpa de dicha situación es la propia obsolescencia de la DMCA, que se ha quedado estancada en un tipo de servidor puro y duro de los de principios de los noventa, sin tomar en cuenta la evolución de dichos servidores hacía empresas verticalizantes y dominantes con contenidos con privacidad abierta. Hay quien apunta que estas facilidades normativas han facilitado el crecimiento de la "Nube", pero también el efecto regresivo sobre otras, en especial, a través de una decisión judicial sobre copyright - Cartoon Network v. Cablevision (2008)—, a parte del cual, según Lerner (2008), se han expandido las inversiones en Internet.

Fruto de la obsolescencia de la normativa, se han promovido en Estados Unidos dos leyes cuya sola proposición ha generado controversia y rechazo total: la "Stop Online Privacy Act", también conocida como "SOPA", por parte de la Cámara de Representantes; y la "Protect Intellectual Properties Act", o conocida como PIPA, instada por el Sanado. La fuerte reacción ha supuesto frenar dichas leyes (Sopa Affects Students, Educators and Libraries, 2011).

Así, Google se ha manifestado en contra de ambas, alegando que se atentaría contra el crecimiento económico y el avance tecnológico. El interés de Google queda aún más claramente reflejado cuando dice que lo que debe existir es una normativa alternativa como la "Open Act", que lo que haría sería impedir el acceso al dinero por parte de las empresas que se lucran de dicha situación irregular o ilegal.

\subsection{En España, pendientes de la eficacia de la Legislación Sinde-Wert}

Antes de la normativa conocida como Ley Sinde-Wert, la jurisprudencia española había tenido la oportunidad de pronunciarse sobre Google y contenidos. Así la Audiencia Provincial de Barcelona estableció en 2008 que:

La creación de una página Web y su introducción en la red responde a la finalidad de divulgarla en ese medio, lo que se logra principalmente gracias al servicio prestado por buscadores como Google, que necesariamente hacen uso del contenido de la página Web y en la medida que lo hacen con la única finalidad de facilitar la labor de búsqueda y discriminación por el internauta de los resultados obtenidos con su solicitud, llevan a cabo un uso social tolerado de aquellas obras, que responde además a la finalidad perseguida por el autor. Esta reflexión viene guiada por el sentido común, que debe impedir sancionar y prohibir una actividad que no sólo no perjudica al titular de los derechos de propiedad intelectual sobre un sitio Web sino que le beneficia, pues contribuye a conseguir una de las finalidades implícitamente perseguidas por el autor, que es su difusión y acceso a los internautas, siendo además efímera e incidental la reproducción y comunicación realizada.

La excepción tecnológica frente al contenido prima en la sentencia antes reproducida. En realidad, la Ley de Servicios de la Sociedad de la Información contiene diversas exenciones de responsabilidad en los arts. 13 y siguientes mera transmisión, caching, alojamiento de datos y provisión de enlaces e instrumentos de búsqueda de contenidos en la red)—, que no se aplican indiscriminadamente a todos los prestadores de servicios de intermediación enumerados en el anexo b), sino en cada caso a los que cumplen con los respectivos presupuestos legales.

En el caso de un buscador como Google, su inclusión en el anexo tiene relación con la previsión contenida en el artículo 17 LSSI, que sí le es de aplicación, aunque resulte irrelevante para este pleito. El art. 17 LSSI no resulta de aplicación al presente caso pues, si bien se refiere a la actividad de los prestadores de servicios "que faciliten enlaces a otros contenidos o incluyan en los suyos directorios o instrumentos de búsqueda de contenidos", lo hace para eximirles de la responsabilidad "por la información a la que dirijan a los destinatarios de sus servicios siempre que se cumplan unas condiciones-, lo que no afecta a la conducta que estamos juzgando que es la copia caché y el ofrecimiento de la misma, al mostrar los resultados de las búsquedas solicitadas".

Hasta el 1 de marzo de este año no entró en vigor la Ley Sinde-Wert, pues le faltaba el Reglamento, pero aún así, se está pendiente de las órdenes ministeriales que indicaran el procedimiento de denuncia. La demora es de más de dos años. Seguramente, lo que va a ocurrir será un acto reflejo de lo ya se ha hecho por parte de Estados Unidos con el Caso Megaupload, es decir, buscar una noticia efectista más que efectiva.

El problema sigue abierto, como muestra realiza en Google la búsqueda "cine". No aparece ninguna web de descarga legal de películas posicionada en primer lugar, pero sí hay CineGratis.net o Cine.com. Es decir, Google promueve y favorece un determinado tipo de resultados que no son desinteresados, sino, más bien todo lo contrario, pues busca masa crítica para su negocio. De forma que la tolerancia y pasividad frente a Google puede afectar al libre mercado legal. 


\section{La "Nube" y el derecho de la competencia: oligarquía dominante liderada por Google}

\subsection{La Oligarquía en el Mercado}

En la actualidad, tanto la Comisión Europea, Dirección de Competencia en Europa, como la Federal Trade Commission en Estados Unidos, están investigando a Google por posibles prácticas anticompetitivas.

En 2011,Google tenía una posición de dominio en el mercado como buscador: mediante ordenador de $65-70 \%$, y en móviles y tabletas el $97 \%$. A su vez había crecido exponencialmente en otros productos y servicios, convirtiéndose en una empresa absolutamente verticalizada: Google Places/Local, Google Finance, Google News, You Tube, Google Travel, Google Maps, Google Flight Search, Google Product Search, Gmail... Su segundo competidor está en las antípodas, digáse Yahoo o Bing.

Los acuerdos que firma Google con terceros son acuerdos donde - sí o sí- tienes que aceptar toda su gama de productos y servicios, lo cual en términos de derecho de la competencia le ha permitido posicionarse de forma vertical, coming full circle, y puede suponer eliminar rivales y limitar la posición del consumidor ante otras ofertas menos agresivas en muchos aspectos (véase Wright, 2011).

A su vez, Apple está también en el punto de mira de las autoridades de la competencia de los Estados Unidos por sus acuerdos de ebooks para su iPad con editoriales y plataformas en línea. A su vez, Apple hace de chivo expiatorio en relación a la integración de Google en el sistema operativo de Apple, por defecto, en toda la gama de productos Apple, es decir, privilegiando productos y servicios de Google frente a los de terceros competidores.

El Departamento de Justicia Americano está valorando la legalidad en términos de derecho de la competencia de la compra por parte de Google de la empresa ITA Software Inc., que ofrece servicios de compra de billetes de avión en línea, por valor de 700 millones de dólares. A la vez, Google ha comprado Motorola Mobility por valor de 12,5 billones de dólares, no solamente por una cuestión de patentes, sino también para seguir ampliando su masa crítica de clientes. Lo mismo acaba de ocurrir con Facebook con la compra de Instagram.
14.2. La definición de producto-servicio y mercado frente a un ser híbrido

El gran problema para analizar Google desde un punto de vista de derecho de la competencia es que es difícil definir su producto-servicio, para luego establecer su verdadera cuota de mercado, como precisa el derecho de la competencia. Google es un ser híbrido: es tanto un buscador como un proveedor de servicios y contenidos en Internet. De hecho, la verticalización de Google se ha producido no solamente por la creación de productos y servicios por parte de Google, sino también por la compra de otras compañías, como por ejemplo DoubleClick.com, en Estados Unidos (1).

Google es una empresa de la nueva economía vertical y digital, que está adquiriendo los rasgos del caso Microsoft. Sin embargo las normas que existen son de la era analógica. De hecho, la Comisión Europea en su comunicado del 30 de Noviembre de 2010, ha definido el mercado de Google como "an online advertising platform in a virtual market place that brings together advertisers and publishers offering advertising space on the Internet" (Casos 1 Plus V y Ciao; Kramler, 2011). Igualmente es interesante, el horizonte que describe Enderle (2012) en Forbes sobre Apple, Google y Microsoft.

La misma línea de interpretación de la Dirección General de la Competencia de la Comisión Europea ha sido desarrollada por el Consejo Nacional de la Competencia Español, en su resolución de 1 de diciembre de 2011:

\footnotetext{
Google está dentro de producto relevante relativo a la publicidad on line: (i) la publicidad on line distinta de la publicidad off line. (ii) Existe cierto apoyo para definir los mercados más estrechos. Estos más estrechos serían la publicidad on line ligada a las búsquedas en Internet, dado que responde a una acción del usuario, y la publicidad on line en los teléfonos móviles, ligado a búsquedas en Internet, ya que diseñan específicamente para este dispositivo y adoptar la forma de breves textos en los que se puede pinchar para ir a la página web del anunciante o para llamarle por el móvil.
}

\subsection{Los resultados de búsqueda de Google} sospechosamente no neutrales

Empresas como Expedia, Yelp y CitySearch se quejan de los supuestos resultados de búsqueda "naturales" de Google — pues no lo son, sino que son interesados- así como de la política de precios de los anuncios. Se trata de saber si Google aumenta deliberadamente las tarifas de publicidad a sus competidores y, en cambio, prioriza los resultados de búsqueda de sus productos y servicios. 
En Francia, 1Plus V, un buscador vertical francés, demandó a Google en la Corte Comercial de París por valor de 421 millones de dólares, alegando que sus sitios web estaban escondidos, "blacklisted", perdiendo mucho tráfico. La compañía francesa acusa a Google de manipular los supuestos resultados de búsquedas "naturales" o "neutrales".

14.4. El decadente Microsoft frente al emergente Google: posibilidad de aplicación de los mismos criterios del Caso Microsoft al Caso Google

La Comisión Europea, Dirección General de Competencia, desde el 30 de noviembre de 2010 está investigando a Google sobre las supuestas búsquedas "naturales", "orgánicas" o "algorítmicas", en base al artículo 102 del Treaty on the functioning of the European Union (TFEU), en un caso en parte promovido por Microsoft así como por asociaciones de editores de toda Europa.

Especular sobre la decisión que tomará la Comisión Europea al respecto de Google es complejo; sin embargo, es evidente que hay muchas concomitancias entre el caso Microsoft y el caso Google, por ello es interesante recordar algunos pasajes de la resolución del caso Microsoft:

[Presencia del producto y servicio: omnipresencia]

El sistema operativo de Windows está presente en cualquier PC dentro de las empresas, los servidores no pueden funcionar correctamente si no hay un verdadero grado de interoperabilidad con Windows.

Por consiguiente, se podría dar una falta de interoperabilidad de los datos y su portabilidad en el caso de Google y sus productos y servicios asociados a datos y contenidos de clientes.

\section{[Vinculación de Productos y Servicios]}

Aunque, generalmente, la estandarización puede suponer determinados beneficios, lo que no puede suponer es imponer de forma unilateral por parte de una empresa en posición de dominio a través de acuerdos de vinculación de productos.

En estos momentos Google está favoreciendo sus propios productos y/o los de sus filiales 0 colaboradores.

Es más, la Comisión Europea está examinando si los acuerdos de publicidad con los anunciantes excluyen a terceros.

\section{Conclusiones a modo de "Knocking on Heaven's Door"}

Como dice J. A. Marina (2012) "ahora comprendemos mejor Descartes, hombre discreto que escribió: "Vive quién vive oculto". Antiguamente, los grandes pozos de conocimiento estaban en los monasterios, con sus bibliotecas y copistas. En la actualidad, las bibliotecas tienen grandes retos: no solamente deben preservar y divulgar el conocimiento, sino también asegurar la privacidad y por ende los contenidos, pues solamente así se podrá seguir enriqueciendo el conocimiento.

Es evidente que el cambio de paradigma va más allá de la legalidad, e incluso de un modus vivendi: Se capitalizan y monetarizan los contenidos y privacidad de terceros en pos del crecimiento tecnológico y con la divisa de que nadie puede pasar por encima de Internet. Los intangibles - privacidad, conocimiento, contenidos...- se diluyen frente a la libertad de empresa y una enmascarada libertad de expresión.

Google es el gran Logos que no tiene necesidad de ley pues él es el todo, es omnipresente y cuenta con el reconocimiento social gracias al postulado de la gratuidad. Es difícil que las autoridades nacionales sin agruparse a nivel internacional y actuar al unísono puedan, sino desconectar a este nuevo HAL 900, al menos controlarlo, en este sentido las instituciones educativas así como bibliotecas tienen mucho que decir y llamar con fuerza a las puertas de la "Nube".

\section{Notas}

(1) La Comisión Europea entendió que el acuerdo de DoubleClick no afectaba al Reglamento de Concentraciones, pero en cambio dejó la puerta abierta a posible vulneración de la Normativa de Protección de Datos (véase European Comission, Case M4731).

\section{Normativa}

\subsection{Unión Europea}

Directiva 2000/31/CE, de 8 de Junio de 2000, sobre Comercio Electrónico.

Tratado sobre el Funcionamiento de la Unión Europea (TFEU). Artículos 101 y 102.

\subsection{España}

Ley 2/2011, de 4 de Marzo, de Economía Sostenible (BOE 5 de Marzo de 2011) (Ley Sinde).

Ley 34/2002, de 11 de Julio, de Servicios de la Sociedad de la Información y Comercio Electrónico y Real Decreto $13 / 2012$ de 30 de marzo por el que se modifica, entre otras normas, la Ley 34/2002, de 11 de julio de Servicios de la Sociedad de la Información y Comercio Electrónico.

Real Decreto 1889/2011, de 30 de Diciembre, por el que se regula el funcionamiento de la Comisión de Propiedad Intelectual, Capítulo Vii, Procedimiento de Salvaguarda de los Derechos de Propiedad Intelectual, Art. 15 y siguientes (Ley Wert). 


\subsection{Normativa y resoluciones de Protección} de Datos en España y Europa

\subsubsection{Binding, Corporate Rules}

WP 155 - Preguntas más frecuentes sobre BCRs.

WP 154 - Cuadro que establece la estructura de las BCRs.

WP 153 - Cuadro que establece la relación de los elementos y principios que deben contener las BCRs.

WP 108 - Modelo de solicitud de autorización de transferencia internacional basada en BCRs en el ámbito del procedimiento coordinado.

WP 107 - Documento sobre la competencia de las Autoridades de Control europeas en el procedimiento coordinado de aprobación las BCRs

WP - 74 - Documento sobre la aplicación del artículo 26.2 de la Directiva 95/46/CE a las BCRs.

Decisión de la Comisión, de 26 de julio de 2000, con arreglo a la Directiva 95/46/CE del Parlamento Europeo y del Consejo, sobre la adecuación conferida por los principios de puerto seguro para la protección de la vida privada y las correspondientes preguntas más frecuentes, publicadas por el Departamento de Comercio de Estados Unidos de América.

Decisión 2001/497/CE, de 15 de junio de 2001, relativa a cláusulas contractuales tipo para la transferencia de datos personales a un tercer país.

Versión consolidada de la Decisión 2001/497/CE, de 15 de junio de 2001, relativa a cláusulas contractuales tipo para la transferencia de datos personales a un tercer país.

Decisión 2002/16/CE, de 27 de diciembre de 2001, relativa a cláusulas contractuales tipo para la transferencia de datos personales a los encargados del tratamiento, establecidos en terceros países. (Queda derogada a partir de 15 de mayo de 2010).

Decisión 2004/915/CE, de 27 de diciembre de 2004, por la que se modifica la Decisión 2001/497/CE, de 15 de junio de 2001.

Decisión 2010/87/UE de la Comisión, de 5 de febrero de 2010, relativa a las cláusulas contractuales tipo para la transferencia de datos personales a los encargados del tratamiento, establecidos en terceros países, de conformidad con la Directiva 95/46/CE del Parlamento Europeo y del Consejo

Lista de entidades estadounidenses adheridas a los principios de "Puerto Seguro". http://www.export.gov/safe harbor.

\section{Referencias}

Agencia Española Protección de Datos (2011). Resolución $\mathrm{R} / / 00896 / 2011$, de 17 de mayo de 2011.

Araiza, Alberto G. (2011). Electronic Discovery in the Cloud. // Duke Law \& Technology Review.

Audiencia Nacional (2012). Auto de 27 de febrero de 2012.

Audiencia Provincial de Barcelona (2008). Sentencia de 17 septiembre de 2008, Secc. 15, AC/2008/1773.

Audiencia Provincial de Madrid (2010). Sentencia de 19 Enero de 2010, Sección n9, JUR/2010//133011.

Carrillo, Marc (2009). El Derecho Al Olvido en Internet, El País, 23 Octubre 2009. http://elpais.com/diario/2009/ 10/23/opinion/1256248805_850215.html

Cloud Security Alliance. https://cloudsecurityalliance.org/.

El Periódico (2012). Yahoo demanda a Facebook por violar patentes sobre privacidad y publicidad. // El Periódico. (13 de marzo de 2012). http://www.elperiodico.com/es/noticias/ciencia-y- tecnologia/yahoo-demanda-facebook-por-violarpatentes-sobre-privacidad-publicidad-1538123.

Ellison, Larry (2008). Oracle OpenWorld. 25 de Septiembre 2008.

Enderle, Rob (2012). What Could Kill Apple, Google or Microsoft?. http://www.forbes.com/sites/robenderle/2012 /01/26/what-could-kill-apple-google-or-microsoft/.

European Comission (2008). Case M4731 Google / DoubleClick. http://ec.europa.eu/comm/competition/mergers/ cases/index/m94.html\#m4731

European Comission (2011). Information Society and Media Directorate-General, 5 diciembre de 2011

Fundación de la Innovación, Bankinter (2010). Se avecinan Nubes Informáticas. 22-33

Gellman, Robert (2009). Privacy in the Clouds: Risks to Privacy and Confidentiality from Cloud Computing. http://www.worldprivacyforum.org/pdf/WPF_Cloud_Priva cy_Report.pdf

Goldner, Matt (2010). Winds of Change: Libraries and Cloud Computing. Online Computer Library Center, Inc. OCLC, 2010.

Indegment of Floyd, de 7 noviembre 2010/2010, EWHC 2011.

Kramler, Thomas (2011). Cloud Computing, From an European Competition Law Perspective European Commission, DG Competition..http://docbox.etsi.org/workshop/ 2011/201109_CLOUD/01_ToolsAndLegalConcepts/EC_ DGCompetition Kramler.p̄df

Kroes en Davos, Neelie (2011). Cloud Computing: Public Consultation Report. 27 de Enero de 2011.

Le Crosnier, Hervé (2008). A l'ère de l'«informatique dans les nuages». // Le Monde Diplomatique août 2008, p.19. Véase igualmente, Cloud Computing: Paradigm shift or just hype? https://www-304.ibm.com/businesscenter/ cpe/html0/158782.htm

Lerner, Josh (2008). The Impact of Copyright Policy Changes on Venture Capital Investment in Cloud Computing Companies http://www.ericsson.com/televisionary/sites/ default/files/Cablevision\%20white\%20paper\%20(11\%20 01\%2011)\%20(2)\%20(2).pdf

Lombarte, Artemi (2010). Punto de Vista: A partir de la Protección de Datos. El Derecho al Olvido y su Protección, Cuadernos de Comunicación e Innovación, Fundación Telefónica, Revista Telos, Octubre-Diciembre 2010.

Malpas, Constante (2011). Cloud-Sourcing Research Collections: Managing Print in the Mass-digitized Library Environment. Dublín, Ohio: OCLC, Research.

Marina, Jose Antonio (2012). ES. // La Vanguardia. (7 abril 2012) 23.

Melzer, Marc Aaron (2011). Copyright Enforcement on the Cloud. 439. http://IPLJ.NET/BLOG/archives/volumexxi /book2

Meuli, Gus; Finn, Caitlin (s. d.). Google: Trust, Choice and Privacy. http://www.ethicapublishing.com/ethical/3CH15.pdf

Multh, Karl T. (2009). Googlestroika: Privatizing Privacy, // Duquesne Law Review. 47, 337-353.

Oregon v. Bellar, 217P, 3d 1094 (Or. App.2009).

Renfrew, Lord Andrew Colin, (2012). Y a qué velocidad! Hoy la digitalización del conocimiento modifica la mente. // La Vanguardia. La Contra. (7 de abril de 2012).

Reuters (2012); 29 de febrero de 2012. Europa investigará la nueva política de privacidad de Google. // Reuters España. (29 de febrero de 2012). http://es.reuters.com/ article/topNews/idESMAE81S08P20120229 
Robinson, William Jeremy (1995). Free at what cost?: Cloud Computing Privacy Under the Stored Communications Act. // The Georgetown Law Journal. 98, 1195-1239

Rosen, Jeffrey (2012). The Right to Be Forgotten. www.stanfordlawreview.org/online/privacy-paradox/rightto-be-forgotten

Sanchati, Rupesh; Kulkarni, Gaurav (2011). Cloud Computing in Digital and University Libraries. // Global Journal of Computer Science and Technology. XI:XII.

SOPA Affects Students, Educators and Libraries (2011) https://www.eff.org/deeplinks/2011/12/how-sopa-affectsstudents-and-educators

Stallman, Richard (2008). Cloud computing is a trap, warns GNU founder Richard Stallman. // The guardian. 29 September 2008. http://www.guardian.co.uk/technology/ 2008/sep/29/cloud.computing.richard.stallman

Teregowda, P.; Urgoankar, B; Giles, C. Lee (2010). Cloud Computing: A Digital Library Perspective. IEEE Cloud.

Unión Europea (2011). Informe a la consulta pública sobre la Nube, de 5 de diciembre de 2011
Vanacek, Jacqueline (2011). ¿Is the cloud the Answer for the European Economy?. // Forbes. (6 December 2011). http://www.forbes.com/sites/sap/2011/12/06/is-cloud-theanswer-for-the-european-economy/

Veoh Case: Universal Music Group, v. Veoh, Case 0955902, 20/12/2012, Nineth Circuit Recisión.

Weber, Ralf H. (2011). Right to be forgotten, more than a Pandora's Box?. // JIPEC.120-124

Week, Linton (2012). Google and Privacy: is it time to Give Up?, $\quad 29-2-2012$ www.npr.org/2012/02/29/147643243/google-wins-hergiving-up-on-privacy

Wright, Josh, (2011). Truth on the Market, Antitrust Remedies, Barnett v. Barnet on Antitrust. // Journal of Law. 10 (May 2011).

Enviado: 2012-04-17. Versión corregida: 2012-08-21. Aceptado: 2012-08-21. 
